https://doi.org/10.7559/gestaoedesenvolvimento.2019.373

Data de receção: 04/04/2019 Data de aceitação: 23/07/2019

\title{
A GESTÃO DE CONFLITOS NAS ORGANIZAÇÕES: CONCEPTUALIZAÇÃO E DIFERENÇAS DE GÉNERO
}

\section{CONFLICT MANAGEMENT IN ORGANIZATIONS: CONCEPTUALIZATION AND GENDER DIFFERENCES}

\author{
Sandrina Sobral ${ }^{1}$ orcid.org/0000-0001-5499-3472 \\ Filomena Capucho ${ }^{2}$ orcid.org/0000-0003-0093-1430
}

\begin{abstract}
Resumo: A forma de perspetivar o conflito tem sofrido significativas alterações com implicações para as organizações, constituindo-se estas em verdadeiras arenas para a sua formação. Por um lado, surge a visão tradicional, onde o conflito era sinónimo de violência, destruição e irracionalidade e, por outro lado, a visão emergente, onde o conflito é sinónimo de produtividade, ao fomentar a criatividade e a inovação. A comunicação surge como causa e solução, em simultâneo, devendo ser instigadas estratégicas ajustadas à natureza e à situação de conflito, baseadas na cooperação entre as partes, naturalmente distintas. Através de uma revisão bibliográfica, objetivou-se refletir o "estado da arte" no âmbito da gestão de conflitos nas organizações, dando ênfase às diferenças de género. Os resultados não evidenciaram um estilo estritamente associado ao género masculino ou ao feminino; contudo, reconhece-se a utilização de uma estratégia de negociação e menos de confrontação pelas mulheres, em oposição aos homens. Identifica-se escassa investigação empírica em Portugal neste domínio, podendo este estudo contribuir no seu progresso, ao estabelecer bases teóricas que a sustentem.
\end{abstract}

Palavras-Chave: conflito, estilos de gestão de conflito, comunicação, género

${ }^{1}$ Mestranda em Gestão na Universidade Católica Portuguesa - Viseu. E-mail: sandrinarsobral@gmail.com

${ }^{2}$ Doutorada em Línguas e Literaturas Modernas, com Agregação em Linguística. Professora da Universidade Católica Portuguesa - Viseu. E-mail: fcapucho@gmail.com 
Abstract: The way that the conflict has been considered has undergone significant changes with implications for the organizations, which become real arenas for their constitution. In one hand, there is the traditional view, where conflict was synonymous of violence, destruction and irrationality, and, on the other hand, the emergent perspective, where conflict is a synonymous of productivity, fostering creativity and innovation. Communication emerges as a cause and as a solution at the same time, and strategies adjusted to the nature and situation of conflict should be instigated, based on cooperation between the different, naturally distinct parties. Based on a literature review, our objective was to reflect about the "state of the art" in the context of conflict management in organizations, with emphasis on gender differences. The results did not show a style strictly associated with the masculine or feminine gender; however, it is recognized that the use of negotiation strategies and less confrontation by women, as opposed to men. There is little empirical research in Portugal in this domain, and this study may contribute to its progress, by establishing the theoretical bases that support it.

Key-words: conflict, conflict management styles, communication, gender

\section{INTRODUÇÃO}

Na década de 1920, autores clássicos e pioneiros da teoria organizacional, como Taylor, Weber e Fayol, entendiam que o conflito era nefasto para a eficiência organizacional, veiculando uma ideia negativa sobre este e suas repercussões. O conflito deveria ser eliminado e, para isso, a hierarquia superior teria de controlar e regulamentar os seus trabalhadores (Bonaldo, Lana, Muller, \& Paines, 2016; Rego, 1997).

Tradicionalmente, a sociedade e, concretamente, as organizações, assentam em valores anti conflito e de evitamento, onde são procuradas incessantemente formas de os vencer, negligenciando eventuais problemas subsequentes, como a perda de confiança e até quebra na relação entre as partes. Porém, o conflito surge como uma inevitabilidade humana, enquanto propriedade da interação (Brahnam, Margavio, Hignite, Barrier, \& Chin, 2005; Caetano \& Vala, 2007; Fatile \& 
Adejuwon, 2011; Ferreira, Neves, \& Caetano, 2011; Reis \& Silva, 2012), e, apesar de ser considerado prejudicial quando em excesso, pode revelarse uma mais-valia quando bem gerido (Milenkovska, Markovska, \& Nikolovski, 2017; Santos \& Abella, 2017).

Numa abordagem emergente, o conflito é tido como fundamental ao desempenho eficaz de um grupo numa organização, existindo um estímulo para a manutenção de um certo nível, despertando a criatividade da equipa (Cunha \& Leitão, 2016; Reis \& Silva, 2012). Assim, uma das questões centrais sobre o tema recai sobre o carácter funcional ou disfuncional do conflito, sendo hoje comummente aceite que o conflito possui aspetos positivos, negativos, funcionalidades, e disfuncionalidades (Cunha \& Leitão, 2016).

A falta de comunicação é apontada como uma das principais causas de conflito. Contudo, por vezes, os conflitos resultam de divergências quanto aos valores, objetivos e interesses e, nestes casos, o incrementar da comunicação pode ampliá-lo (Ferreira, Martinez, Nunes, \& Duarte, 2015; Neves, Garrido, \& Simões, 2015). Nesta medida, o conflito pode ser facilitador da comunicação (efeito positivo e construtivo) e, conjuntamente, pode gerar insegurança comunicacional (efeito negativo e destrutivo) (Cunha \& Leitão, 2016).

Na opinião de Neves, Garrido e Simões (2015, p.211), "gerir conflitos nas organizações é, antes de mais, aceitar e gerir a diferença”. Neste âmbito, podem ser enquadradas as diferenças de género na gestão e resolução de conflitos. Trata-se de uma temática com reconhecido interesse e importância, pois as diferenças individuais e de personalidade têm influência no estilo utilizado para lidar com o conflito (Chusmir \& Mills, 1989; Neves et al., 2015).

São contempladas várias formas de resolução de conflitos, geralmente utilizadas de forma dinâmica. Uns querem sempre ganhar, outros deixam ganhar, outros procuram a solução ótima, outros tentam evitá-lo, outros tentam acomodar-se. Alguns estudos sugerem que as mulheres apresentam maior tendência que os homens na adoção do estilo de compromisso (Holt \& DeVore, 2005), noutro os homens utilizam o estilo de competição e as mulheres o estilo de evitamento (Brewer, Mitchell, \& Weber, 2002), noutros nem foram encontradas diferenças de género (Mcintyre, 2007). 
Face ao exposto, objetiva-se analisar a produção científica, por forma a refletir o estado da arte, recorrendo a uma metodologia de pesquisa bibliográfica. O artigo inicia com a conceptualização do conflito e suas dimensões, identificação de possíveis causas e estratégias de gestão e resolução. Adicionalmente, procura-se perceber o papel da comunicação enquanto causador/solucionador de conflito, bem como conhecer as bases teóricas que sustentam as eventuais diferenças de género na forma de lidar com situações conflituosas. Seguidamente, é apresentada uma revisão de estudos empíricos, de modo a expor a forma como a temática tem vindo a ser cientificamente estudada. Por fim, são apresentadas as considerações finais, limitações do presente estudo e recomendações para trabalhos futuros.

\section{O CONFLITO: CONCEITO, DIMENSÕES E CAUSAS}

A crescente investigação científica direcionada às Ciências Organizacionais tem sido relevante para a pluralidade na conceptualização do conflito (Dimas, Lourenço, \& Miguez, 2005), não subsistindo uma definição simplista do conceito (Mcintyre, 2007).

Segundo Reis e Silva (2012), o conflito pode ser entendido como um processo de discórdia entre duas ou mais partes, tanto ao nível do indivíduo como do coletivo, ou seja, indivíduos, grupos, departamentos ou organizações, sustentado pelas suas ações, interceções e reações. Por outras palavras, o "conflito é definido como um processo interativo manifestado em incompatibilidade, desacordo ou dissonância dentro ou entre entidades sociais, i. e., indivíduo, grupo, organização, etc." (Blake \& Mouton, 1981, p.29).

Nesse sentido, com base na literatura, podem listar-se quatro principais formas de incompatibilidades:

1. Incompatibilidade de interesses e preocupações: o conflito pode ser encarado como um desacordo em relação a interesses ou ideias, no qual um interveniente percebe que os seus interesses são opostos ou negativamente afetados pelo outro interveniente (Slabbert, 2004; Wall \& Callister, 1995).

2. Incompatibilidade nas metas e valores: o conflito é visto como uma interação de intervenientes interdependentes que concebem uma oposição 
de objetivos, metas e valores, onde a outra parte é vista como potencial influenciador (John \& Christine, 1999).

3. Incompatibilidade na interação: designadamente da interação humana no contexto laboral entre intervenientes, onde a execução de determinadas tarefas por uma das partes depende das escolhas, decisões e comportamentos da outra (Fatile \& Adejuwon, 2011).

4. Incompatibilidade na relação: a interdependência dos indivíduos numa organização pressupõe um elevado nível de cooperação entre os seus intervenientes; porém, surgem desentendimentos pessoais e os conflitos ocorrem (Fatile \& Adejuwon, 2011).

$\mathrm{Na}$ visão tradicional, os conflitos eram percebidos com algo nefasto, devendo ser evitado sempre que possível (Lima, 2014; Reis \& Silva, 2012). Não obstante, há quem veja a existência de conflitos como um processo intrínseco à dinâmica organizacional (Alves et al., 2012; Fatile \& Adejuwon, 2011), podendo daí resultar ganhos significativos para as organizações (Dimas, Lourenço, \& Miguez, 2005; Milenkovska, Markovska, \& Nikolovski, 2017; Santos \& Abella, 2017).

Rego (1997) considera que devem ser balanceados certos aspetos negativos e positivos do conflito. Pode ser benéfico, quando moderado e bem gerido, ao encorajar novas ideias impulsionadoras de mudança e a inovação, ao promover o envolvimento da equipa, ao gerar diversidade de pontos de vista, ao motivar os envolvidos à compreensão das posições contrários e até ao fortalecer as relações na organização. Contudo, pode gerar consequências negativas, como a criação de um ambiente organizacional desagradável, desgaste emocional com repercussões evidentes no quotidiano e o aprofundamento das diferenças, obstaculizando a comunicação, cooperação e interajuda.

A tipologia do conflito assenta essencialmente na natureza das interações estabelecidas e na intensidade como se desenvolvem. Deste modo, o conflito pode ser denominado, conforme a sua origem, a nível intrapessoal ou interno, interpessoal, interorganizacionais e intraorganizacional. Os conflitos internos ou interpessoais ocorrem no próprio indivíduo quando existe divergência de ideias ou pensamentos, podendo tratar-se de um conflito repulsão-atração (rejeição de uma alternativa por seleção de outra), repulsão-repulsão (escolha mediante duas alternativas desagradáveis) ou ainda atração-repulsão (escolha entre 
alternativas vantajosas e desvantajosas, situação mais comum). A nível interpessoal sucede com indivíduos dentro da organização, podendo estar na origem diferenças de personalidade ou discordância comportamental e cultural do grupo e da organização. Por último, a nível organizacional pode ocorrer entre organizações (interorganizacionais), na procura de clientes por exemplo, ou dentro da própria organização (intraorganizacional), abrangendo a generalidade das partes (Rahim \& Bonoma, 1979; Reis \& Silva, 2012; Torres, 2012).

Importa ainda distinguir conflitos funcionais de disfuncionais. No primeiro caso, atua de forma construtiva no grupo melhorando o seu desempenho, podendo ser do tipo de tarefa ou de processo. O tipo de tarefa está relacionado com o conteúdo e os objetivos de trabalho, estimulando a discussão e troca de ideias entre o grupo, enquanto o tipo processo remonta à forma de execução do trabalho, reforçando a afetação de disponibilidade de tempo quando o nível de conflito é reduzido. Já o conflito de relacionamento, refere-se às relações interpessoais, impacta negativamente sob desempenho do grupo, ao diminuir a compreensão mútua, aumenta o desentendimento entre os envolvidos e limita a execução de tarefas desenvolvidas na organização (Neves et al., 2015; Reis \& Silva, 2012).

Neste sentido, para a compreensão dos dois polos - funcional e disfuncional, há que avaliar o nível de motivação dos envolvidos, isto é, se ambos estão direcionados para a sua resolução positiva (Alves et al., 2012). A diferença reside no valor ético, suportado no "maior bem para o maior número" (Deutsch, 1969, p.10). Paralelamente, é fundamental considerar o contexto comportamental em que os intervenientes se enfrentam e as suas condutas durante o conflito (Cunha \& Leitão, 2016).

Lima (2014) distingue os conflitos segundo quatro níveis de intensidade, passíveis de auxiliar no desenvolvimento apropriado e implementação de estratégias de intervenção. São eles: (1) conflito latente: quando não existe a consciência pelos intervenientes da sua existência; (2) conflito percebido: quando existe a consciência da existência de conflito pelos envolvidos, embora não manifestado; (3) conflito sentido: quando existe um manifesto envolvimento emocional, levando as partes envolvidas a agir de forma competitiva; (4) conflito 
manifesto: percebido pelos envolvidos e por terceiros, podendo interferir na dinâmica organizacional.

Assim, a tabela I resume os diversos tipos de conflito consoante a sua origem, intensidade e efeito.

\section{Tabela I}

Tipos de conflitos

\begin{tabular}{|c|c|c|}
\hline Âmbito & Tipos de conflitos & Referências \\
\hline Quanto à origem & $\begin{array}{l}\text { - Intrapessoal ou } \\
\text { interno } \\
\text { - Interpessoal } \\
\text { - Interorganizacional } \\
\text { - Intraorganizacional } \\
\end{array}$ & $\begin{array}{lr}\text { Rahim e } & \text { Bonoma } \\
\text { (1979); Reis e Silva } \\
(2012) ; \\
\text { (2012) }\end{array}$ \\
\hline $\begin{array}{l}\text { Quanto à } \\
\text { intensidade }\end{array}$ & $\begin{array}{l}\text { - Latente } \\
\text { - Percebido } \\
\text { - Sentido } \\
\text { - Manifesto } \\
\end{array}$ & Lima (2014) \\
\hline Quanto ao efeito & $\begin{array}{l}\text { - Funcional } \\
\text { - Tarefa } \\
\text { - Processo } \\
\text { - Disfuncional } \\
\text { - Relacionamento }\end{array}$ & $\begin{array}{l}\text { Ma et al. (2017); } \\
\text { Neves et al. (2015); } \\
\text { Reis e Silva (2012) }\end{array}$ \\
\hline
\end{tabular}

Fonte: elaboração própria

São apontadas inúmeras causas para a existência de conflitos (Wall \& Callister, 1995). Para Reis e Silva (2012), as situações que podem estar na origem do conflito resumem-se a três categorias: em primeiro lugar, falhas de comunicação por uma interpretação deficiente, linguagem incompreensível, características específicas de um grupo ou a alteração de informação transmitida; em segundo lugar, a estrutura organizacional, quanto existe diferente distribuição dos objetivos entre indivíduos; por último, questões pessoais como a existência de personalidades distintas. De facto, num estudo efetuado por Neves, Garrido e Simões (2015), foram identificadas como principais causas de conflito numa organização a falta de comunicação, a falta de planeamento das tarefas diárias, o ambiente 
organizacional, a qualidade da relação e aspetos emocionais, tanto pelo superior hierárquico como pelos subordinados.

\section{COMUNICAÇÃO E CONFLITO}

Aspetos ligados à comunicação são considerados a principal causa de conflito (Neves et al., 2015; Reis \& Silva, 2012). Contudo, sob outra perspetiva, a comunicação pode revelar-se um meio de solucionar o conflito, através da utilização combinada de competências individuais (Neves et al., 2015).

Na literatura, é apontada maior eficácia na gestão e resolução de conflitos quando são utilizados simultaneamente múltiplos canais de comunicação, pelo maior rigor na transmissão de intenções, expetativas e mensagens simultâneas. Porém, a interação face a face promove mais a cooperação quando comparada com a comunicação escrita, verbal ou visual, onde as partes tendem a focar-se nas questões originárias de conflito, negligenciando os ganhos mútuos e privilegiando o confronto. Nesse sentido, surgem duas táticas de comunicação: a ameaça e a promessa. Na primeira são movidas estratégias comunicativas no sentido de convencer o outro que será alvo de punição. Já a promessa é destinada a convencer a outra parte, que receberá uma recompensa (Caetano \& Vala, 2007).

Por outro lado, a escuta ativa e a promoção do feedback, no sentido de reduzir eventuais distorções e lapsos na transmissão da mensagem (nas quais o processo de comunicação é fértil), e a argumentação construtiva e persuasiva são apresentadas como instrumentos importantes na gestão eficaz do conflito (Ferreira et al., 2015). Neves et al. (2015) sugerem quatro dimensões importantes para a contextualização das competências pessoais comunicativas, particularmente, a identificação da origem e do conflito, descurando divergências quanto a eventuais crenças pessoais; a dissociação entre a parte conflituosa e o conflito; a transformação do conflito num problema passível de resolução e a capacidade de aceitar as diferenças e não limitar a situação a estritamente certa ou errada.

Assim, a forma como se comunica pode ampliar ou atenuar o nível de conflito. A escolha das palavras a transmitir deve ser ponderada por forma a não gerar desentendimentos no processo de perceção individual do 
sentido da mensagem. A simples forma de tratamento assume significados diferentes de acordo com o grupo social, podendo ser interpretadas de forma cordial ou hostil (Neves et al., 2015).

Em suma, uma comunicação eficaz não implica, per si, a resolução das situações conflituosas. Porém, comunicar eficazmente e ter a perceção de que está a ser entendido facilita significativamente a resolução construtiva do conflito (Deutsch, 1990), transformando as acusações em sentimentos, a culpa em causas e as "verdades" em "perceções", aceitando que " diferença é apenas uma diferença, não certa ou errada" (Ferreira et al., 2015, p.386).

\section{A GESTÃo de CONFLITO}

Na verdade, muitas organizações veem com desconfiança a existência de conflitos internamente, culpando-se em parte pela sua existência. Ainda predomina a ideia que o conflito revela falta de cooperação e solidariedade, levando à negação da existência com receio de ser visto como uma situação negativa (Mcintyre, 2007).

De facto, como já referido anteriormente, os conflitos são uma realidade, quer seja no âmbito pessoal quer profissional, não devendo ser necessariamente reduzidos ou eliminados, mas sim geridos (Rahim \& Bonoma, 1979). Deste modo, destaca-se a importância da gestão eficiente e eficaz do conflito, de modo a proporcionar resultados construtivos nas organizações (Fatile \& Adejuwon, 2011; Lima, 2014). Na opinião de Rahim (2002), para que a gestão de conflitos seja eficaz, devem ser tidas em conta três premissas fundamentais: a utilização de estratégias orientadas para o pensamento crítico e inovador e para uma eficácia organizacional sustentável; a satisfação das necessidades e expetativas dos stakeholders estratégicos e o comportamento ético do gestor na definição do problema.

\subsection{Estilos de gestão de conflito}

Uma primeira abordagem envolveu uma dicotomia para a resolução de conflitos - a cooperação e a competição. A cooperação pretende o estabelecimento de uma relação positiva entre as partes, enquanto a competição pode traduzir uma relação de interdependência que pode 
revelar-se negativa (Deutsch, 1990). Nesse sentido, um processo cooperativo é caracterizado por uma comunicação aberta e honesta entre os intervenientes, em contraste, um processo competitivo é caracterizado por falta de comunicação ou comunicação falaciosa (Deutsch, 1994). Contudo, nesta abordagem não figurava a diversidade de comportamentos dos envolvidos, sendo apontada como principal crítica por alguns autores (Van de Vliert \& Kabanoff, 1990).

Putnam e Wilson (1982, citados por Cunha, 2008; Cunha \& Leitão, 2016; Torres, 2012) propõem três estilos de resolução de conflitos: a não confrontação, com a cedência ou afastamento por uma das partes; foco na solução mutuamente desejável com um acordo ou concessão na busca do compromisso e controlo por via da única e exclusiva preocupação dos interesses próprios, menosprezando a outra parte em conflito.

Por sua vez, surgem três abordagens que procuraram a interação dos interesses individuais através de um modelo bidimensional, originando cinco estilos possíveis para lidar com conflitos, amplamente destacados na literatura (Van de Vliert \& Euwema, 1994).

A abordagem proposta por Rahim e Bonoma (1979) procura equilibrar a preocupação com a satisfação dos próprios interesses e da outra parte envolvida. A sua combinação resulta em cinco estilos específicos (Rahim, 2002; Rahim \& Bonoma, 1979):

- Evitamento (também designado por inação): reduzida preocupação com o interesse próprio e com o outro, onde é ignorada a existência de conflito e evitada a aproximação a outros que podem causálo. Esta estratégia surge adequada a situações de conflito preliminares.

- Acomodação (também designado por obediência, cedência ou submissão): reduzida preocupação com o interesse próprio e elevada com o outro, imprimindo esforços para a resolução da situação de conflito a fim de satisfazer a outra parte. Esta estratégia é útil quando não existe familiarização com o assunto gerador de conflito, quando uma das partes está disposta a desistir, esperando uma retribuição da outra parte e quando se está perante uma parte mais fraca e se pretende preservar o relacionamento.

- Integração (também designado por cooperação ou resolução de problemas): elevada preocupação com o interesse próprio e com o outro, onde a resolução passa pela discussão e resolução dos pontos de vista em 
divergência em benefício de ambas as partes, sendo considerado o estilo mais eficaz. É particularmente útil quando só uma parte não pode resolver o conflito, como em situações envolvendo questões estratégicas de longo prazo numa organização.

- Dominação (também designado por competição ou confrontação): elevada preocupação com o interesse próprio e reduzida com o outro, refletindo um comportamento de egoísmo ao fazer sobrepor os seus interesses aos da parte oposta, fomentando ainda mais conflitos no futuro. É útil para um superior hierárquico na comunicação de medidas menos consensuais ou quando um subordinado é demasiado assertivo.

- Compromisso: traduz a intenção de satisfação de ambas as partes através de cedências mútuas, o tradicional dar e receber. É considerado uma mais-valia quando as partes conflituantes são igualmente poderosas e quando se pretendem evitar conflitos prolongados.

À semelhança, Thomas (1976, citado por Thomas, 1988), apresenta o cruzamento de duas dimensões, a assertividade (nível de satisfação dos interesses próprios) e cooperação (nível de satisfação dos interesses do outro). A articulação destas dimensões pode resultar em competição (assertividade e não-cooperação), evitamento (não assertividade e não cooperação), colaboração (assertividade e colaboração), acomodação (cooperação e não assertividade) e compromisso (assertividade e cooperação médias).

Esta é encarada na literatura como a abordagem mais completa; ainda assim é-lhe apontada uma limitação pelo facto de não equacionar a complexidade do comportamento humano, circunscrita aos cinco estilos (Cunha \& Leitão, 2016).

Numa abordagem mais recente, Van de Vliert e Euwema (1994), definiram como eixos bidimensionais o nível de atividade e o nível de agradabilidade, com alguma complexidade (Cunha, 2008) . Neste modelo são apostos dois comportamentos de conflito, a cooperação (agradável) e a competição (desagradável). Na cooperação, a atividade resulta na negociação podendo originar compromisso ou foco na solução e, a inatividade, resulta no não-confronto, podendo gerar o evitamento (desagradável) ou acomodação (agradável). Ao contrário, na competição, a atividade resulta num confronto direto, podendo originar um problema em confronto (não desagradável) ou ultrapassar o confronto 
(desagradável), e a inatividade resulta no confronto indireto, podendo gerar o controlo do processo (não desagradável) e resistência (desagradável) (Van de Vliert \& Euwema, 1994).

Assim, não existe a utilização de uma estratégia dominante na gestão individual de conflitos; porém, é necessário que as partes consigam adotar a estratégia mais adequada à natureza do conflito e à sua contingência, exigindo flexibilidade pessoal (Neves et al., 2015).

\subsection{Diferenças de género}

Uma primeira perspetiva surge nos anos 70 e 80, na área da Psicologia, onde foram estudadas as diferenças de género baseadas nos traços de personalidade. Desse modo, desenvolveram-se pesquisas no sentido de apurar se as mulheres eram mais cooperativas e menos interessadas nos ganhos próprios que os homens (por exemplo, Walters, Stuhlmacher, \& Meyer, 1998). Contudo, não foram encontradas evidências que confirmassem esta predição teórica, chegando mesmo a observarem-se resultados contraditórios (Cunha, 2008).

Uma perspetiva secundária, emerge da necessidade do género feminino afirmar o seu papel no campo da negociação, existindo uma representação estereotipada aplicada ao negociador. Deste modo, o procedimento masculino constitui a norma e o procedimento feminino é concebido como diferente, o que perpetua a hierarquia relacional. Apesar de ser mote de vários estudos, é considerado preliminar para a compreensão do papel do género na negociação do conflito; contudo, podem ser destacadas algumas bases teóricas que tentam articular o género e a negociação (Cunha, 2008; Monteiro, 2010).

A primeira é a socialização sustentada principalmente em duas premissas: as mulheres têm mais habilidade verbal que os homens: estando associados melhores resultados em tarefas onde a comunicação verbal seja uma realidade e, os homens são mais agressivos: tanto fisicamente como verbalmente (Maccoby \& Jacklin, 1974, p.351-352), o que é um fator facilitador na exigência de valores económicos (Cunha, 2008).

A segunda perspetiva é a existência de diferenças quanto aos valores morais, desenvolvida por Gilligan (1982), evocando-se duas principais orientações. Por um lado, a perspetiva da moralidade masculina baseada 
na justiça, no respeito pelos direitos individuais e pelas normas universais e, por outro lado, a perspetiva da moralidade feminina, assente na "moral de cuidado", impondo às mulheres o cuidado dos outros. Neste sentido, a conceção baseada na relação com os outros é apelidada de uma perspetiva conservadorista, ao promover a submissão das mulheres na sociedade e ao predomínio de estereótipos sociais, particularmente de que a mulher nasceu para cuidar dos outros (Kuhnen, 2014).

A terceira ilustra a existência de diferenças de género genéricas numa situação de negociação, explicadas essencialmente em três domínios, designadamente a socialização, construção da identidade e valores morais, anteriormente expostos (Cunha, 2008).

Uma quarta perspetiva remete ao autoconceito, baseada nos discursos culturais, nas organizações sociais e na psicologia individual, apoiando a sobreposição do poder masculino e opressão das mulheres (Bem, 2008). Por um lado, as mulheres veem as negociações como uma componente de relação adotando um comportamento mais flexível que o género oposto, dando uma impressão mais positiva que os homens e, por outro lado, percecionam a negociação como tipicamente praticada por homens, que adotam um estilo de confrontação (Cunha, 2008). Por outras palavras, o autoconceito leva à adoção de comportamentos baseados na perceção, as mulheres percecionam uma relação de dependência face aos outros e os homens consideram-se independentes.

Por último, são apontadas as diferenças de género quanto ao comportamento do oponente, partindo do pressuposto de comportamentos de género idênticos. As diferenças são motivadas essencialmente por fatores como as expectativas formadas pelas partes e a discriminação sustentada numa "atitude cavalheiresca" em favor do género feminino (Cunha, 2008). Dentro desta orientação teórica, reconhecem-se três potenciais fatores de diferenças de género, designadamente: (1) expectativa: a violação das expetativas do outro provoca maior preocupação para as mulheres do que para os homens; (2) discriminação: diferenças entre géneros baseadas nas expectativas antecipadamente formadas e (3) desconstrutivismo: associado a um critério de desempenho que é parcialmente contra as mulheres e consequentemente intensifica as diferenças de género (Monteiro, 2010). 
Assim, a tabela II resume as diversas perspetivas e respetivos domínios tentando fornecer uma explicação quanto às diferenças de género na gestão e resolução de conflitos.

\section{Tabela II}

Perspetivas teóricas da relação entre o género e a gestão de conflitos

\begin{tabular}{|c|c|c|}
\hline $\begin{array}{c}\text { Perspetivas } \\
\text { teóricas }\end{array}$ & Domínios & Referências \\
\hline Socialização & & Cunha (2008) \\
\hline Valores morais & $\begin{array}{l}\text { - Justiça } \\
\text { - "Moral de cuidado" }\end{array}$ & $\begin{array}{l}\text { Gilligan (1982) e } \\
\text { Kuhnen (2014) }\end{array}$ \\
\hline $\begin{array}{l}\text { Diferenças } \\
\text { genéricas }\end{array}$ & $\begin{array}{l}\text { - Socialização } \\
\text { - Construção de identidade } \\
\text { - Valores morais } \\
\end{array}$ & Cunha (2008) \\
\hline Autoconceito & $\begin{array}{l}\text { - Discursos culturais } \\
\text { - Organizações sociais e } \\
\text { psicologia social } \\
\end{array}$ & $\begin{array}{l}\text { Bem (2008) } \\
\text { Cunha (2008) }\end{array}$ \\
\hline $\begin{array}{l}\text { Comportamento } \\
\text { do oponente }\end{array}$ & $\begin{array}{l}\text { - Expetativas } \\
\text { - Discriminação } \\
\text { - Desconstrutivismo }\end{array}$ & $\begin{array}{l}\text { Cunha (2008) e } \\
\text { Monteiro (2010) }\end{array}$ \\
\hline
\end{tabular}

Fonte: elaboração própria

$\mathrm{Na}$ literatura, surgem alguns estudos empíricos relacionados com as diferenças de género nos estilos adotados perante uma situação de conflito.

Monroe, Disalvo, Lewis e Borzi (1990) levaram a cabo uma investigação para examinar a relação entre o género e os estilos de resolução conflito de supervisores em relação a subordinados, numa amostra de 381 elementos (207 homens e 174 mulheres). Concluíram que o género do subordinado exerce influência na escolha do estilo de gestão em comparação com o género do supervisor. Particularmente, os subordinados homens empregarem mais estilos de confrontação quando os seus supervisores são mulheres e as mulheres subordinadas empregam mais frequentemente o estilo de evitamento com supervisores do género masculino.

Com o intuito de examinar os estilos de conflito dos gerentes 
masculinos e femininos, considerando a dualidade de papéis na sociedade - a nível organizacional e a nível familiar, Chusmir e Mills (1989) recorreram a uma amostra de 201 gerentes ( 99 homens e 102 mulheres). Neste contexto, ambos os géneros tendem a lidar de uma forma mais competitiva no trabalho do que em casa e usam o estilo de acomodação mais frequentemente em casa do que no trabalho.

Monteiro (2010) questionou 537 indivíduos (designadamente, estudantes, administrativos e negociadores), concluindo que o género feminino apresenta uma relação positiva com estratégias orientadas para a solução de problemas, como a integração e o compromisso. Verificouse, também, maior eficácia negocial, assente em características como a sensibilidade e interdependência, tipicamente associadas ao género feminino, facilitadoras na compreensão dos interesses do outro. Por sua vez, os homens apresentam uma maior tendência na utilização de estratégias competitivas como a dominação, tendencialmente menos eficaz em termos de negociação.

Sutschek (2002), a partir de uma amostra de 74 elementos constituída por 29 homens e 45 mulheres, encontrou diferenças de género significativas na utilização da estratégia compromisso. Porém, os homens obtiveram resultados ligeiramente superiores nas estratégias acomodar e dominar e as mulheres nas estratégias evitar e integrar. O estudo constatou ainda que as mulheres usavam as estratégias de conflito pela seguinte ordem: integrando, comprometendo, obrigando, evitando e depois dominando, e os homens nesta ordem: integrando, comprometendo, obrigando, dominando e evitando, diferenciando apenas nas duas últimas estratégias.

Analogamente, Rahim (1983) também encontrou diferenças em lidar com situações conflituosas, em que as mulheres utilizam mais o estilo integrar, evitar e compromisso e menos de acomodar, em comparação com o género oposto. Também, Chanin e Schneer (1984) encontraram o estilo integrar como o preferido pelas mulheres. Já McIntyre (2007) verificou a estratégia compromisso como dominante para o género feminino; ainda assim, relativamente às demais estratégias, a autora não encontrou divergências entre género. Por seu turno, Brewer, Mitchell e Weber (2002), para além de estudar a relação entre género e estratégias utilizadas, incluíram o estatuto organizacional. Os resultados revelaram 
que os homens utilizam o estilo competitivo e as mulheres o evitamento. Além disso, os indivíduos com um estatuto organizacional elevado recorriam mais ao estilo integrativo, enquanto com estatuto organizacional baixo reportaram maior uso dos estilos evitação e acomodação.

Numa investigação desenvolvida por Brahnam, Margavio, Hignite, Barrier e Chin (2005), com 163 estudantes de cursos de sistemas de informação, os resultados evidenciaram que as mulheres são mais propensas a utilizar um estilo de colaboração e os homens são mais propensos a evitar conflitos. Baseada na suposição teórica de que a colaboração tem efeitos mais produtivos e a evitação é mais perturbadora, os autores afirmam que o género feminino pode possuir qualidades que tornam mais eficaz a resolução de conflitos.

Num outro estudo com académicos de uma Universidade Libanesa, foi evidenciada a utilização do estilo de competição pelo género masculino e de colaboração pelo género oposto. Adicionalmente, os autores verificaram diferenças significativas entre géneros nos estilos competição e compromisso (Kaseh, 2014).

Tendo em conta as inconsistências nos resultados observadas em várias investigações, Holt e DeVore (2005) publicaram uma meta-análise, com 36 estudos, investigando as diferenças entre os estilos de gestão de conflito, género, estatuto organizacional e cultura. De entre os vários resultados alcançados destaca-se que as mulheres empregam tendencialmente mais o estilo compromisso que os homens, independentemente da cultura; em culturas individualistas os homens usam mais o estilo de dominação e o estilo compromisso é mais utilizado pelas mulheres; e, os homens utilizam mais frequentemente o estilo de dominação do que as mulheres na relação com os seus superiores hierárquicos. Estes resultados coincidem, em parte, com os de Thomas, Thomas e Schaubhut (2008), em que os homens exibiam o estilo dominação.

Herk, Thompson, Thomas e Kilmann (2011) analisaram as diferenças de género na gestão de conflitos considerando uma amostra internacional representativa, composta por 6.168 indivíduos de 16 países (não se inclui Portugal). Foram encontradas diferenças estatisticamente significativas entre homens e mulheres nos cinco estilos de conflito, sendo que o estilo 
competição foi o que mais diferiu entre países, por género, tendencialmente mais utilizada pelos homens.

Em suma, e atendendo aos estudos revisados sobre a relação entre os estilos de gestão de conflito e género, destaca-se a divergência nos resultados alcançados. Em primeiro lugar, podem estar na origem diferenças nos instrumentos utilizados, na amostra considerada ou nos contextos investigados. Em segundo lugar, esta divergência pode ser explicada com base na existência de distintos fatores, passíveis de influenciar os resultados, nomeadamente o papel na sociedade e o contexto (Chusmir \& Mills, 1989), os estereótipos de género (Deutsch, 1990, 1994), características de personalidade (Monteiro, 2010) e o status organizacional (Brewer et al., 2002; Monroe et al., 1990).

\section{CONCLUSÃO}

Num momento de profunda mudança e transformação nas organizações, com uma envolvente dinâmica assente na forte concorrência, é essencial a busca de soluções alternativas de gestão e resolução de conflitos, otimizando tempo e recursos. Numa visão emergente, a gestão eficaz de conflitos pode trazer ganhos significativos na criatividade e inovação (Santos \& Abella, 2017), estimulando o crescimento profissional da equipa e consequentemente da organização (Dimas et al., 2005).

Para este estudo, foi nosso objetivo analisar as particularidades da gestão de conflito nas organizações, identificando eventuais causas, dimensões e estratégias para a sua gestão e resolução, nomeadamente ao nível das estratégias individuais numa perspetiva de diferenças de género.

Verificou-se que a gestão de conflitos é uma temática ainda pouco explorada em Portugal, à semelhança do constatado por Mcintyre (2007); contudo, quando analisada a diferença de género, esta escassez ainda é mais notada. Esta tendência poderá dever-se a dois motivos: por um lado, a descrição e a explicação das diferenças de género assentes em insuficiente fundamentação quanto às causas, por outro lado, a possível perceção de certos investigadores acerca da reduzida importância das diferenças de género, enfatizando outras variáveis consideradas relevantes, como referido por Monteiro (2010). 
A comunicação e a capacidade de relacionamento com os outros são características amplamente associadas maioritariamente ao género feminino, consideradas essenciais na estimulação da resolução de situações conflituosas. Foram apontadas diversas estratégias adotadas consoante o género tendo por base vários estudos científicos, revelando, no entanto, uma certa inconsistência nos resultados alcançados, ainda que sobressaia a utilização de uma estratégia de negociação com menor confrontação pelo género feminino, em oposição ao género masculino.

Apresenta-se como principal limitação do presente estudo a inexistência de uma componente empírica que promova a discussão dos resultados, passível de proporcionar uma ligação em contexto real à componente teórica revisada.

Espera-se com este trabalho, através da uma análise dos escritos de revisão teórica e empírica, fomentar futuras investigações por forma a diagnosticar quais as estratégias individuais de resolução de conflitos mais utilizadas pelos líderes nas organizações, atendendo a eventuais diferenças de género, assim como perceber quais os estilos comportamentais apropriados a diferentes situações conflituosas.

\section{BIBLIOGRAFIA}

Alves, A. C., Miranda, F., Pinto, J., Santos, L., Matos, V., \& Magalhães, J. (2012). A importância do conflito organizacional: identificação estratégias e modelos. PSIQUE, 8, 113-137.

Bem, S. L. (2008). The lenses of gender: transforming the debate on sexual inequality. New Haven, CT, US: Yale University Press. Disponível em:

https://books.google.pt/books?id=3pTX1VIAwS0C\&dq=The+lenses +of+gender:+Transforming+the+debate+on+sexual+inequality.\&hl= pt-PT\&sa=X\&ved=0ahUKEwj5i76Nr5ncAhWMwBQKHSbAHUQ6AEIKzAA

Blake, R. R., \& Mouton, J. S. (1981). Management by Grid ® Principles or Situationalism: Which? Group \& Organization Studies, 6(4), 439455.

Bonaldo, S. A., Lana, R. D., Muller, F. M., \& Paines, P. A. (2016). As organizações como fonte de conflitos de poder. Revista Da 
Administração UFSM, Santa Maria, 9(3), 506-519.

Brahnam, S. D., Margavio, T. M., Hignite, M. A., Barrier, T. B., \& Chin, J. M. (2005). A gender-based categorization for conflict resolution. Journal of Management Development, 24(3), 197-208.

Brewer, N., Mitchell, P., \& Weber, N. (2002). Gender role, organizational status, and conflict management styles. International Journal of Conflict Management, 13(1), 78-94.

Caetano, A., \& Vala, J. (2007). Gestão de recursos humanos (3ª). Lisboa: Editora RH.

Chanin, M. N., \& Schneer, J. A. (1984). A study of the relationship between jungian personality dimensions and conflict-handling behavior. Human Relations, 37(10), 863-879.

Chusmir, L. H., \& Mills, J. (1989). Gender differences in conflict resolution styles of managers: at work and at home. Sex Roles, 20(34), 149-163.

Cunha, P. (2008). A diversidade de práticas na relação entre género, conflito e negociação. Revista Faculdade de Ciências Humanas e Socias da UFP, 5, 266-279. Disponível em:

https://bdigital.ufp.pt/bitstream/10284/898/1/266-279.pdf

Cunha, P., \& Leitão, S. (2016). Manual de gestão construtiva de conflitos ( $3^{\text {a }}$ Ed). Porto: Edições Universidade Fernando Pessoa.

Deutsch, M. (1969). Conflicts: Productive and Destructive. Journal of Social Issues, 25(1), 7-41.

Deutsch, M. (1990). Sixty years of conflit. International Journal of Conflict Management, 1(3), 237-263.

Deutsch, M. (1994). Constructive Conflict Resolution: Principles, Training, and Research. Journal of Social Issues, 50(1), 13-32.

Dimas, I. D., Lourenço, P. R., \& Miguez, J. (2005). Conflitos e desenvolvimento nos grupos e equipas de trabalho - uma abordagem integrada. Psychologica, 38, 103-119.

Fatile, J. O., \& Adejuwon, K. D. (2011). Conflict and conflict management in tertiary Institutions: the case of nigerian universities. European Journal of Humanities and Social Sciences, 7(1).

Ferreira, I. A., Martinez, L. F., Nunes, F. G., \& Duarte, H. (2015). Gestão de recursos humanos para gestores ( $\left.1^{\mathrm{a}}\right)$. Lisboa: Editora $\mathrm{RH}$.

Ferreira, J. M. C., Neves, J., \& Caetano, A. (2011). Manual de 
psicosociologia das organizações. Lisboa: Escolar Editora.

Gilligan, C. (1982). In a different voice: psichological theory and women's development. Consultado a 12-07-2018 em:

https://books.google.pt/books?id=OujZ0iSyNWgC\&printsec=frontco ver $\& d q=I n+a+$ different + voice $\&$ hl $=$ pt-

PT\&sa=X\&ved=0ahUKEwi91 tKh2JfcAhWEbxQKHdNVDKAQ6A EIKDAA\# $\mathrm{v}=$ onepage $\& \mathrm{q} \& \mathrm{f}=$ false

Herk, N. A., Thompson, R. C., Thomas, K. W., \& Kilmann, R. H. (2011). International Technical Brief for the Thomas-Kilmann conflict mode instrument. CPP, Inc. Disponível em:

http://www.kilmanndiagnostics.com/sites/default/kilmann-private10020393424lksdjfksdjfl093u/TKI_International_Brief.pdf

Holt, J. L., \& DeVore, C. J. (2005). Culture, gender, organizational role, and styles of conflict resolution: A meta-analysis. International Journal of Intercultural Relations, 29(2), 165-196.

John, R. D., \& Christine, E. F. (1999). Conflict management across cultural boundaries: a case analysis from a multinational bank. European Business Review, 99(6), 383.

Kaseh, O. F. El. (2014). Gender effect on the styles of managing interpersonal conflicts: an exploratory study on the academic staff of Sirte University. Journal of Basic and Applied Scientific Research, 4(5), 191-203.

Kuhnen, T. A. (2014). A ética do cuidado como teoria feminista. Anais do III Simpósio Género e Políticas Públicas, (2004), 1-9. Disponível em: http://www.uel.br/eventos/gpp/pages/arquivos/GT10_Tânia

Aparecida Kuhnen.pdf

Lima, N. M. (2014). Gestão de conflitos nos jogos de empresas. Revista LAGOS - UFF, 2(2), 58-72.

Ma, L., Yang, B., Wang, X., \& Li, Y. (2017). On the dimensionality of intragroup conflict: An exploratory study of conflict and its relationship with group innovation performance. International Journal of Conflict Management, 28(5), 538-562.

Maccoby, E. E., \& Jacklin, C. N. (1974). The psychology of sex differences. California: Stanford University Press. Disponível em: https://books.google.pt/books?id=6hzsWwz1 aLAC\&printsec $=$ frontco ver $\& \mathrm{hl}=\mathrm{pt}-\mathrm{PT} \# \mathrm{v}=$ onepage $\& \mathrm{q}=$ aggressive $\& \mathrm{f}=$ false 
Mcintyre, S. E. (2007). Como as pessoas gerem o conflito nas organizações : Estratégias individuais negociais. Análise Psicológica, 2 (XXV), 295-305.

Milenkovska, V., Markovska, M., \& Nikolovski, L. (2017). Promotion : Women Leadership and Alternative Methods for Resolving Business Conflicts and Changes in the organization. UTMS Journal of Economics, 8(2), 183-194.

Monroe, C., Disalvo, V. S., Lewis, J. J., \& Borzi, G. (1990). Conflict behaviors of difficult subordinates: Interactive effects of gender. Southerm Communication Journal, 56(1), 12-23.

Monteiro, A. P. (2010). Eficácia negocial e caraterísticas pessoais dos negociadores. Tese de doutoramento. Universidade de Santiago de Compostela. Disponível em:

https://minerva.usc.es/xmlui/bitstream/handle/10347/3007/97884988 75966_content.pdf?sequence $=1$

Neves, J. G., Garrido, M. V., \& Simões, E. (2015). Manual de competências pessoais, interpessoais e instrumentais ( $\left.3^{\mathrm{a}} \mathrm{Ed}\right)$. Lisboa: Edições Sílabo.

Rahim, A., \& Bonoma, T. V. (1979). Managing organizational conflict: a model for diagnosis and intervention. Psychological Reports, 44(3c), 1323-1344.

Rahim, M. A. (1983). A measure of styles of handling interpersonal conflict. Academy of Management Journal, 26(2), 368-376.

Rahim, M. A. (2002). Toward a theory of managing organizational conflict. The International Journal of Conflict Management, 13(3), 206-235.

Rego, A. (1997). Liderança nas organizações: teoria e prática ( $\left.1^{\mathrm{a}} \mathrm{Ed}\right)$. Aveiro: Universidade de Aveiro.

Reis, F. L. dos R., \& Silva, M. J. R. (2012). Princípios de Gestão (1 $\left.{ }^{\mathrm{a}} \mathrm{Ed}\right)$. Lisboa: Edições Sílabo, Lda.

Santos, L. carvalho dos, \& Abella, N. T. T. (2017). Gestão de conflitos organizacional. Revista Multidebates, 1(2), 219-225.

Slabbert, A. D. (2004). Conflict management styles in traditional organisations. Social Science Journal, 41(1), 83-92.

Sutschek, L. B. (2002). Conflict resolution style and experience in management: moderating the effects of gender. VUW-La Crosse JUR, 
5, 69-78. Disponível em:

http://murphylibrary.uwlax.edu/digital/jur/2002/sutschek.pdf

Thomas, K. W. (1988). The conflict-handling modes: toward more theory. Management Communication Quarterly, 1(3), 430-436.

Thomas, K. W., Thomas, G. F., \& Schaubhut, N. (2008). Conflict styles of men and women at six organization levels. International Journal of Conflict Management, 19(2), 148-166.

Torres, S. C. (2012). Estilos de gestão construtiva de conflitos numa organização empresarial. Universidade Fernando Pessoa. Faculdade de Ciências humanas e sociais. Porto.

Van de Vliert, E., \& Euwema, M. C. (1994). Agreeableness and Activeness as Components of Conflict Behaviors. Journal of Personality and Social Psychology, 66(4), 674-687.

Van de Vliert, E., \& Kabanoff, B. (1990). Toward Theory-Based Measures of Conflict Management. Academy of Management Journal, 33(1), 199-209.

Wall, J. A., \& Callister, R. R. (1995). Conflict and Its Management. Journal of Management, 21(3), 515-558.

Walters, A. E., Stuhlmacher, A. F., \& Meyer, L. L. (1998). Gender and negotiator competitiveness : a meta-analysis. Organizational Behavior and Human Decision Processes, 76(1), 1-29. 